\title{
Michel Meslin, L'homme et le religieux. Essai d'anthropologie
}

Préface d'Yse Tardan-Masquelier, Paris, Ếditions Honoré Champion, 2010, 222 p.

\section{Daniel Vidal}

\section{(c) OpenEdition}

\section{Journals}

Édition électronique

URL : http://journals.openedition.org/assr/22926

DOI : $10.4000 /$ assr.22926

ISSN : $1777-5825$

Éditeur

Éditions de l'EHESS

Édition imprimée

Date de publication : 31 décembre 2011

Pagination : 227

ISBN : 9782713223273

ISSN : 0335-5985

Référence électronique

Daniel Vidal, « Michel Meslin, L'homme et le religieux. Essai d'anthropologie », Archives de sciences sociales des religions [En ligne], 156 | octobre-décembre 2011, document 156-80, mis en ligne le 16 février 2012, consulté le 21 septembre 2020. URL : http://journals.openedition.org/assr/22926 ; DOI https://doi.org/10.4000/assr.22926

Ce document a été généré automatiquement le 21 septembre 2020

(c) Archives de sciences sociales des religions 


\section{Michel Meslin, L'homme et le religieux. Essai d'anthropologie}

Préface d'Yse Tardan-Masquelier, Paris, Ếditions Honoré Champion, 2010, 222 p.

Daniel Vidal

\section{RÉFÉRENCE}

Michel MesLIN, L'homme et le religieux. Essai d'anthropologie, Préface d'Yse Tardan-

Masquelier, Paris, Ếditions Honoré Champion, 2010, 222 p.

De l'étude des premiers âges chrétiens à l'anthropologie de l'homme romain, de l'histoire des religions à leur « science ", de l'expérience du divin à l'histoire de la prière, Michel Meslin a composé un ensemble de recherches centrées sur l'avènement, dans la conscience collective et personnelle, de la question du religieux. Moins, doit-on préciser, de la religion comme instance, ou institution, de croyance, que comme expérience subjective, et bouleversante, d'une transcendance. Rendre compte de l'assentiment qui est ainsi donné à telle « découverte ", ou "rencontre ", au plus intime, au plus ultime, de la conscience, comprendre en quoi telle « notion » apparaît à l'auteur décisive dans la constitution de l'être humain en sa singularité, mais aussi bien, et par là-même, en sa relation à autrui, son éthique, etc. - cela requiert un regard anthropologique capable d'accéder au plus près de la formulation du rapport qui lie l'homme à cet absolu, cet impossible nom de Dieu. Impossible, et cependant pensable. L'anthropologie religieuse, que propose M. Meslin en cet ouvrage posthume, est ainsi fondée, abolis tout artifice ou commodité de pensée, sur la mise à nu de ce qui est le plus secret en l'homme, et le plus socialement légitimé.

2 Pour en venir à cette netteté du regard, il fallait d'abord, toute compétence avérée, marquer les limites de ce que la sociologie classique identifiait comme «faits religieux ", situés historiquement, qui prenaient sens à partir de leur inscription dans des ensembles institutionnels, économiques, culturels, au détriment de leur capacité à 
valoir comme signatures de spiritualité et marque originelle de subjectivité. H. Cohen, mais aussi bien Ernst Troeltsch, avaient noté cette difficulté majeure de la prise en compte du religieux comme " fait » historique, à « définir le concept de religion». Un changement de paradigme s'impose, un changement de dispositif de connaissance. Audelà et en deçà du « fait social », il convient de « revenir à la chose même telle qu'elle se présente à la conscience ». Seule, en effet, une phénoménologie est apte à décrire «les vécus de la conscience", en leur complexité, leurs paradoxes, leurs contradictions, leurs cheminements - leurs aboutissements. Et à comprendre, au plus près de leur " vérité ", leurs significations. L'analyste n'est pas extérieur à « l'objet » qu'il analyse : H. R. Jauss définit cette démarche herméneutique par l'aptitude «à se laisser prendre, com-prendre, dans le mouvement et la production de sens » que tel objet livre à qui s'offre à lui. M'approprier un sens «qui m'est d'abord étranger»: voilà la neuve condition d'un savoir exact. Complicité entre deux regards? Sans doute. Mais qui ne signifie nullement que la démarche signe la destitution de l'analyste, et sa fusion/ confusion avec les interprétations qui lui viennent de cet ailleurs si familier. Que M. Meslin soit homme de croyance, de ce point de vue, importe peu: il lui suffit de maitriser les impératifs de la distinction des modes de connaissance qu'impose toute herméneutique, pour donner de l'expérience intérieure du religieux une présence sans ambiguïté.

Dans ce cadre épistémologique, M. Meslin revisite les «notions fondamentales» de la "question religieuse ». Relève-t-elle de la loi naturelle, qui fonderait la religion sur l'universalité de la raison, ou de la loi divine, éclairant cette raison par la Révélation? La loi, en judaïsme, est fondée sur le principe d'Alliance. Ne pas s'y conformer est acte concret et personnel de désobéissance à Dieu, mais non péché "issu d'une faute originelle ». De même en islam, qui définit Dieu comme Loi, et le rapport à l'être humain comme pacte originel. Rompre le pacte n'est pas pécher, c'est subvertir le contrat, dont le jugement dernier soldera le compte. Le christianisme introduit la notion de péché à partir de la thématique du mal, ce mésusage, dans le monde réel, de la liberté naturelle, que Paul identifie à une blessure faite au cœur de la loi divine. Un mal originel, un mal «essentiel ", en ce qu'il serait définition même de ce qui est humain en l'être. Mais la notion de mal comme « désordre » se relie, au plus profond de la conscience chrétienne, au principe de responsabilité. La théodicée chrétienne, rappelle M. Meslin, «a lié l'exercice de la liberté à la souffrance et au mal ». D'Augustin à J. Maritain, en un raccourci saisissant, le mal est défaillance fondamentale de la nature humaine ; plus encore, le mal ne peut se définir comme une "essence », mais une " absence d'être ». Un mystère, disent Gabriel Marcel et Hans Jonas. Qui suppose le retrait de Dieu, que Lévinas dit inexplicable. S'en tenir là, et vivre cependant, c'est faire appel à la foi, cette grâce divine qu'invoquait Luther. En ces trois monothéismes, toute distinction assumée, il est clair que la " présence » de Dieu au centre de la conscience institue (paradoxalement?) le croyant comme sujet responsable, là de son insoumission, ici, de sa «malédiction».

De son « hérésie » ? De ses dérivations, note M. Meslin, remontant à la source du mot. C'est dire que toute religion porte en elle la tentation du multiple, cette œuvre diabolique, contre l'harmonie du symbole et de la tradition. Et ceci, en judaïsme, est pris en charge et décharge. Soit le Talmud de Babylone. De la Bible, est-il écrit, « on ne peut refuser à aucun texte son sens littéral » - mais est légitime la pluralité des significations de cette lettre même : «car il y a soixante-dix visages dans la Torah ». Et chaque interprétation a valeur d'actualisation : qui dit le sens dit aussitôt le temps où 
ce sens se propose. À l'aube du christianisme, point d'orthodoxie première, et pour cause: le "message révélé " est occasion de présentations diversifiées, sinon divergentes. La tradition qui finira par s'imposer ne pourra jamais ignorer, en ses marges, et dès le début, ce que M. Meslin décrit comme « une sorte de pénombre, une frange d'opinions toujours mouvante ». De cette «fluidité ", la tradition viendra, que l'on pourrait entendre comme "développement des virtualités contenues dans l'Écriture ». Il en va de la genèse de la tradition, comme des représentations culturelles et religieuses que M. Meslin interroge une nouvelle fois. Questions de l'origine : la tradition suppose une vaste aventure de l'esprit autour d'un foyer de signes sans intelligibilité immédiate qui fasse décision. A fortiori le thème de "l'origine de l'humain». Vitalisme? Mais d'où, l'énergie qui le sous-tend? Des «sociétés traditionnelles» disent la naissance comme double entrée/sortie : sortie d'un monde en deçà, entrée dans le monde des vivants ; et la mort, sortie du monde des vivants / entrée dans le monde de l'au-delà. Le temps s'écoule sur le mode d'une éternelle mutation, de porte en porte, de passage en passage. Mais l'existence sociale requiert qu'en cette impermanence demeure un principe de stabilité: ce sera le nom, qui instaure/restaure la relation aux ancêtres. Relation/religion. Par ailleurs, si le rôle des religions est bien, comme le souligne $\mathrm{M}$. Meslin, de "réduire l'altérité sexuelle en majorant l'un des deux genres ", masculin ou féminin, c'est de tenir d'emblée la femme pour figure même de cette altérité. Sans doute établit-on l'équation symbolique entre fécondité de la femme et fécondité de la terre, principe de vie et de reproduction, mais site de souillure et de maléfice - Pandora et Eve, emblèmes de malédiction, signatures du «mal». Majorant cependant en la femme la raison de fécondité et de survie de l'espèce, J.-J. Bachoffen avait développé l'hypothèse du matriarcat comme "structure sociale originelle sublimée en religion", car fondée sur le "mystère de la vie». M. Meslin s'écarte de cette conception de la Terre-Mère, qui relèverait des religions polythéistes, quand les monothéismes privilégieraient le principe "masculin », Dieu le Père. Sans pour autant méconnaître toutes les interprétations, notamment jungiennes, concernant la figure « maternelle » de Dieu.

5 Il est des paganismes, il est des monothéismes, des déismes, des syncrétismes, et toutes autres modalités concevables de croyances. Peut-on dès lors parler d'un universel religieux, d'un homo religiosus, qui définirait l'être humain comme par " loi naturelle » habité de transcendance? C'est de son expérience de la nature que découlerait, selon Frazer, la religion "vécue par l'homme». Ce n'est que déporter le problème, en affectant à la nature un coefficient fondamental de sacralité. Sur ce thème, M. Meslin conteste les théories de Mircea Éliade qui, instituant la nature comme site du sacré, conçoit celui-ci comme paradigme transhistorique : le réel est le sacré. Du cosmique à l'ontologique, l'assimilation s'effectue aussitôt. Mais il n'est alors pour l'être humain nulle quête qui vaille, puisque le principe de transcendance est immanent aux choses, telles quelles apparaissent à son regard et au profond de son existence. C'est bien ce qui pose problème à l'auteur : «ce sont les hommes, et eux seuls, qui sont la mesure de la sacralité des êtres et des choses, parce qu'ils sont les agents de leur sacralisation ». Il était essentiel, pour M. Meslin, de rapatrier en effet au profit de l'existence humaine le pouvoir de définir, dans l'univers de la nature et des vivants, ce qui relève du profane, et ce qui abonde au plan du sacré. Ce plan lui-même implique un rapport au divin, qui ne saurait être «le pur produit d'une hypothèse empirique». Mais d'une expérience conduite au vif de la conscience, en son vécu le plus singulier, qui est sa chance et son défi. 
6 Dans la mouvance de P. Ricœur, pour qui «la pensée sur le "transcendantal" passe par l'anthropologique», et ouvre sur un être "marqué par une finitude qu'il ne peut dépasser ", définissant ainsi le "site " même de Dieu en cet au-delà de la "fin ", M. Meslin ne conçoit pas que le religieux puisse apparaître «dans le processus de dévoilement de soi ", mais dans le mouvement qui en appelle au principe d'altérité : Dieu est cet Autre, ce "Tout-Autre", qui est condition du sujet. L'auteur sollicite K. Barth: "Dieu est la transcendance intérieure de l'homme et le monde sa transcendance extérieure ", et M. Buber : «"Je" a besoin d'un "Tu" éternel ». Mais de cette altérité fondatrice, l'être humain est éminemment responsable. Ruse de la «raison religieuse»? Meslin: «Le fondement de toute religion est une expérience existentielle dans laquelle l'être humain se construit par rapport à un Dieu, et où il est à la fois créateur et créature responsable de son nouvel être ». Convenons cependant que « Dieu », ce sans nom et sans lieu, n'est jamais « déjà là », en instance de dévoilement, mais, altérité absolue, ce qui, dans l'intimité la plus secrète, se propose comme principe permanent de dépassement. De transgression. S'est-on tant éloigné de l'homme, que l'on ne puisse en lui seul fonder la raison de Dieu?

7 Seule alors une anthropologie relieuse est en effet capable de rendre compte de ce qui s'institue comme religions, en leurs variations extrêmes, et leur profil commun. Et de rouvrir le débat sur les conditions d'apparition des monothéismes, matrice générale à tout culte ancien polythéiste, ou phase ultime de l'«universalisation» de la pensée religieuse. On peut regretter, à cet égard, que l'auteur n'ait pas fait référence à Durkheim ou M. Augé, pour tenter de régler la question. Mais lui savoir gré de lire, dans les grands récits mythiques autour desquels s'organise en toute société le champ religieux, non point des textes rigides, mais des textes que la transmission orale ne cesse de modifier, introduisant ainsi au cœur de toute tradition un principe d'incertitude. Car il n'est point de texte archétypal, original, qui puisse, si l'on peut dire, faire foi. Ainsi en va-t-il de la divination, cette «interrogation de l'accidentel», assez ouverte à interprétations pour qu'elle «n'occulte pas la responsabilité de l'être humain». "L'éclatement du croire», que M. Meslin identifie dans nos sociétés rationnelles-modernes, participe de cette remise de l'homme au centre de l'expérience religieuse, qui est dès lors revendiquée comme expérience vécue. «Pour tout croyant, écrit M. Meslin, une évidence : c'est l'existence d'une relation entre l'homme et un Absolu transcendant qui constitue l'aspect duel du phénomène religieux ». Au-delà de ce réquisit, c'est la catégorie d'intériorité qui est en jeu, cet espace d'intimité où se formule l'exigence incontournable d'altérité. La «rencontre de Dieu», qui s'y énonce, peut se dire inscription, à même son corps et esprit, de cet «autre » sans qui nul ne saurait être humain. Il est remarquable, note l'auteur, que Dieu, en la Torah, se dise paix et équité, shalom. Trois lettres font décision : slm disent aussi bien islam. De cette prise en compte de l'altérité, une éthique procède, condition du droit, condition de soi. Mais un soi assez creusé de temps multipliés, pour n'exister véritablement que comme "défaillance perpétuelle ", selon la lumineuse formule de Fénelon. C'est en ce « milieu incompréhensible entre le néant et l'être » que l'anthropologie religieuse doit pourtant saisir l'être humain, son existence "en religion", sa croyance et sa foi. Mais son incroyance aussi bien, et sa raison. 